\title{
Substitution Arg140Gly in HA/H7 attenuated highly patho- genic avian influenza virus FPV/Rostock/34 (H7N1)
}

\author{
Anastasia Treshchalina ${ }^{1}$, Yulia Postnikova ${ }^{1}$, Elizaveta Boravleva ${ }^{1}$, Alexandra Gambaryan ${ }^{1 *}$, Alla Belyakova ${ }^{1}$, Aydar \\ Ishmukhametov', Galina Sadykova², Alexei Prilipov², Natalia Lomakina². \\ 1 Chumakov Federal scientific center for the research and development of immune-and-biological products. , \\ Village of Institute of Poliomyelitis, Settlement "Moskovskiy", 108819, Moscow, Russian Federation; nar- \\ moriel5991@gmail.com (A.T); postni.yulya@ya.ru (Y.P,). e@boravlev.mccme.ru (E.B.); al.gam- \\ baryan@gmail.com (A.G.), \\ 2 The Gamaleya National Center of Epidemiology and Microbiology of the Russian Ministry of Health, Mos- \\ cow, 123098, Russian Federation; gksadykova@gmail.com (G.S.); a_prilipov@mail.ru (A.P.); \\ nflomakina@gmail.com (N.L.) \\ * Correspondence: e-mail: al.gambaryan@gmail.com Tel.: +7-985-136-3586
}

\begin{abstract}
The H7 subtype of avian influenza viruses (AIV) stands out among other AIV. H7 viruses circulate in ducks, poultry, equine and have repeatedly caused outbreaks of disease in humans. In order to study the pathogenicity factors of H7N1 viruses, several laboratory variants of the A/FPV/Rostock/34 (H7N1) strain were obtained by passages in the chicken lungs. After 10 such passages, a variant was obtained that differed from the parent virus by amino acid substitutions Val109Phe in PB2, Gln621Lys in PB1, Thr32Ala and Leu586Phe in PA Gly140Arg in HA1 and Ala101Thr in HA2 (numbering by H3), Ser82Arg in M2, Arg118Lys and Met124Arg in NS1. No differences were found in proteins NA, NP, M1 and NS2. The resulting variant was hundreds of times more pathogenic for chickens than the original laboratory variant of the virus. The study of intermediate passages showed that the jump in pathogenicity occurs sharply between the fifth and sixth passage through the chicken lungs. By cloning these variants, a pair of strains (R5p and R6p) were obtained, and the complete genomes of these strains were sequenced. Single amino acid substitution was revealed, namely Gly140Arg in HA1. It is important to emphasize that this substitution is a reversion, since Arg is located in position 140 HA1 of original the A/FPV/Rostock/34 (H7N1) virus (GenBank). This amino acid is located at the head part of the hemagglutinin, adjacent to the receptor-binding site. In addition to the increased pathogenicity, R6p differs from R5p by an increased affinity for a negatively charged receptor analogue, an increased affinity for MDCK cells, while maintaining a receptor specificity profile.
\end{abstract}

Keywords: highly pathogenic avian influenza viruses, pathogenicity factors.

\section{Introduction}

The main hosts of influenza viruses (IV) H1 - H6, H8 - H12 and H14 are wild ducks, while separate evolutionary branches have formed in other hosts [1]. The H13 and H16 subtypes are primarily gull viruses. The H7 subtypes and the phylogenetically close H15 also stand out among avian influenza viruses (AIV). Although they actively circulate in both ducks and poultry, evolutionary trees show that chickens are the primary hosts, and ducks are secondary hosts of H7 viruses [2]. H7 viruses in ducks are generally completely apathogenic and lack a polybasic cleavage site. However, they easily introduced into the poultry population and rapidly evolved with increasing pathogenicity [3]. Numerous outbreaks of the H7N2 virus occurred in 1996 in Pennsylvania. Despite the absence of a polybasic cleavage site, the virus caused economic losses of the order of several million USD. Later, viruses of this evolutionary branch caused outbreaks in Miami (2001) and Virginia (2002) [4]. Outbreaks of the H7N4 virus with RKRKRG cleavage sites were recorded in Australia in 1997 [5]. 
More than 500 outbreaks caused by the H7N1 virus took place in Italy in 1999-2000. At first the proteolytic site did not contain the sequence of positively charged amino acids, while by the end of this period the presence of a "furin site" became a characteristic feature of these viruses [6].

A powerful epizootic of the H7N7 virus swept in 2003 in the Netherlands, Belgium and Holland. This epizootic became famous as infection with this virus was noted in 83 people, of whom one died [7].

In 2013, the first human cases of the H7N9 virus were detected in China. Since then, laboratory confirmed about 1,600 cases of which 615 are fatal. An intriguing circumstance was that human diseases occurred without concomitant outbreaks in poultry [8]. Over the past 6 years, H7N9 infections have occurred continuously, and the virus have accumulated mutations that change receptor specificity and increase drug resistance [9]. In 2017, a variant of the H7N9 virus with a polybasic cleavage site appeared [10]. H7N9 infection in humans are now under control by closing markets and vaccinating poultry in many provinces. Meanwhile, the H7N9 virus continues to shed from the environment and from wild birds, making new outbreaks likely. There were suspicions that the virus was transmitted sporadically from human-to-human. However, its ability to transform into a strain with regular human-to-human transmission remains unknown. The high (about 40\%) mortality from the H7N9 virus remains the most dramatic factor. H7N9 vaccines for humans are currently in clinical trials. However, they are not on the market. Under these conditions, monitoring wild and migratory birds can help to prevent the spread of the virus. [11]. The study and tracking of mutant forms with increased pathogenicity and/or altered receptor specificity is also an important element of pre-pandemic preparation. The pathogenicity of the virus for mammals is influenced by substitutions in the PB2 and PA genes. Thus, the substitutions of Glu358Val in PB2 and Pro190Ser and Gln400Pro in PA attenuate the virus for mice and reduce the inflammatory process after infection [12]. It has been shown that substitutions at positions 111, 146 and 340 of hemagglutinin $\mathrm{H} 7$ increase the pathogenicity and transmissibility of the virus [13]. A single substitution of Gly228Ser in H7 HA affects the tissue tropism of the virus and dramatically increases the affinity for human tracheal cells [10].

Chicken Plague Virus A/FPV/Rostock/34 (H7N1) is a highly pathogenic avian influenza virus (HPAIV) that caused outbreaks in chickens in the thirties of the twentieth century. The main determinant of the high pathogenicity of HPAIV is the polybasic cleavage site of hemagglutinin (HA), which is cleaved by furins - (intracellular serine proteases of animal cells). This type of cleavage allows the virus to multiply in the internal organs and leads to fatal systemic infection, in contrast to local infection caused by low pathogenic influenza viruses due to the limited cleavage of $\mathrm{HA}$ at monobasic cleavage sites. The study of the spread of the A/FPV/Rostock/34 virus in the host organism and tissue tropism showed that the virus caused a generalized infection, which was strictly limited to endothelial cells in all organs. Endotheliotropism is determined, on the one hand, by the cleavability of HA by furins, which ensures the penetration of the virus into the vascular system, and, on the other hand, by the polarity of virion budding and the pattern of receptor expression, which prevents the spread of infection into the tissues surrounding the endothelium [14].

The strong dependence of the pathogenicity of viruses on the structure of the cleavage site made it possible to construct attenuated variants with a shortened or altered cleavage site, which can serve as live vaccines or producers of inactivated vaccines against avian influenza viruses of the H7 subtype [15].

Another way to attenuate $\mathrm{H} 7$ viruses is to obtain temperature-sensitive mutants that multiply only at low temperatures. Corresponding mutations are usually located in one of three regions of the HA: (i) at the end of the alpha helix, (ii) between the globular region and the stem, and (iii)) in the basal stem domain. These regions are critical for HA assembly and hence transport, there as that mutants cannot leave the endoplasmic reticulum at non-permissive temperatures and are incorrectly trimerized $[16,17]$. The Ile512Leu mutation in PB2 also led to temperature sensitivity and attenuation [18]. 
H7 viruses differ from other AIVs not only in their epidemiology, but also in the structure of the receptor-binding site (RBS). In HA H7, the constant for all subtypes Pro185 is replaced by Ser. The residues at positions 185-189 of H7 HA have small side chains (usually Ser-Gly-Ser-Thr-Thr), while other avian viruses besides Pro185 have at least one large amino acid residue at these positions. As a rule, Lys or Arg are located in 193 positions of the RBS in H7 HA, which is also typical for chicken H5N1 viruses. The positively charged amino acid residue 193 provides binding of the negatively charged sulfo group of the Neu5Ac@2-3Gal@1-4-(6-O-Su)GlcNAc@-(Su3'SLN) and Neu5Ac@2-3Gal@1-4(Fuc@13)(6-Su)GlcNAc (Su-Slex) receptors [2]. Another characteristic property of H7 HA is a pair of arginines: 140 and 141. Of which Arg141 is constant for H7 HA, while Arg140 in rare cases is replaced by Lys or a small amino acid. The study of the causal relationship between the structural features of the RBS H7 and the biology of these viruses is the goal of this work. We compared two variants of the influenza A/FPV/Rostock/34 (H7N1) virus differing in the amino acid at position 140 of HA1 (Gly or Arg) and investigated the mechanism of increasing pathogenicity associated with this mutation.

\section{Materials and Methods}

\subsection{Reagents}

Fetuin and horseradish peroxidase were from Serva, Switzerland. Antibodies against mouse and chicken immunoglobulins conjugated with horseradish peroxidase were from (Sigma, USA). Sialylglycopolymers were from GlycoNZ (Auckland, New Zealand).

\subsection{Viruses.}

The attenuated laboratory strain A/FPV/Rostock/34 was obtained from the collection of the Gamaleya National Center of Epidemiology and Microbiology. Non-pathogenic mallard virus A/mallard Sweden 91/02 (H7N9) was kindly provided by Dr. R. Fouchier (Department of Virology, Erasmus Medical Center, the Netherlands). Passage variants of A/FPV/Rostock/34 were obtained in this study. Ten-day-old embryonated chicken eggs (CE) were inoculated with $10^{2} \mathrm{EID}$ of viruses, incubated at $36^{\circ} \mathrm{C}$, monitored and cooled immediately after death or after 60 hour of incubation. Infectious allantoic fluids (IAF) were harvested and tested by hemagglutination assay. The virus amount was expressed in hemaglutinating units (HAU). 50\% infective dose (EID 50 ) for each virus stock was determined by titration in CE.

\subsection{Animals}

Chickens and embryonated chicken eggs were purchased from State poultry farm "Ptichnoe" (Moscow, Russia). All studies with HPAIV viruses were conducted in a biosafety level 3 containment facility. BALB/c mice (weight in the range from 8 to $10 \mathrm{~g}$ ) were purchased from "Lesnoye" farm, Moscow, Russia.

\subsection{Ethics Statement}

Studies involving animals were performed in accordance with the (European Convention for the Protection of Vertebrate Animals used for Experimental and Other Scientific Purposes, Strasbourg, 18 March 1986). All appropriate measures were taken to ameliorate animal suffering. 94 chickens were used in the study; 39 chickens survived and were subsequently kept in the bird housing facility for repeated detection of antibody levels. 55 chickens were humanely euthanized after they showed signs of severe disease. The study design was approved by the Ethics Committee of the Chumakov Federal scientific center for the research and development of immune-and-biological products, Moscow, Russia (Approval \#4 from 2 December 2014). 


\subsection{Sequencing}

Viral RNA was isolated from the allantoic fluid of infected chicken embryos with a commercial QIAamp Viral RNA mini kit (Qiagen, \# 52904). Full-length viral genome segments were obtained by revers transcription and PCR with specific terminal primers [19], MMLV and Taq-polymerase (Alpha-Ferment Ltd., Moscow). The amplified fragments were separated by electrophoresis in 1-1.3\% agarose gel and subsequently extracted from the gel with the Diatom DNA Elution kit (Isogen Laboratory Ltd., Russia, \# D1031). Sequencing reactions were performed with terminal or internal primers [20] with the BrightDye TM Terminator Cycle Sequencing Kit v3.1 (Nimagen, the Netherlands), followed by analysis on an ABI PRISM 3100-Avant automated DNA sequencer (Applied Biosystems). The Lasergene software package (DNASTAR, Inc.) was used for assembly and analysis of nucleotide sequences. The complete genomes were sequenced for the original strain and its variant obtained after 10 passages in the chicken lungs, as well as cloned variants of R5p and R6p. Their Accession Numbers in GenBank are MT914267-MT914274, MT916934-MT916941, MT916987-MT916994, MT917013MT917020.

\subsection{Infection of chickens}

7-day-old or 56-day-old chickens were infected intranasal with undiluted allantoic fluid containing the tested viruses. All birds were assessed daily for body weight, clinical signs of disease and mortality. On day 3 post-infection, lungs of one chick from each group were taken for virus determination.

\subsection{Passaging the virus through chicken lungs}

For the first passage, four 7-day-old chickens were infected intranasal with undiluted virus-containing IAF (100 $\mu \mathrm{L}$ per chicken). On days 3 after infection, one chick were humanely euthanized; lungs were excised, homogenized, and centrifuged. The rest chicks were monitored for signs of disease. The lung supernatant was used for infection of the new group of chicken. In order to study the passage variants of the virus, the CE was infected with lung homogenates and IAF was used to analyze the viral genome, to determine receptor specificity, virulence, and for other purposes.

\subsection{Assessment of pathogenicity index}

The intravenous pathogenicity index (IVPI) was carried out with accordance to the recommendation of the World Organization for Animal Health [21]. Six weeks old specific pathogen-free chickens (SPF) with no previous history of vaccination against influenza virus were used. Chickens were injected intravenously into a wing vein, using $0.2 \mathrm{~mL}$ of an inoculum containing 1:10 dilution (using sterile PBS) of IAF. Birds were examined twice daily for up to 10 days. At each observation, each bird was scored 0 if normal, 1 if sick, 2 if severely sick, 3 if dead. If birds were too sick to eat or drink, they were humanely sacrificed and scored as dead at the next observation. Dead birds were assigned a score of 3 daily, up to the tenth day of the experiment. The scores for all birds in the group were summed daily, and then summed up over all 10 days of observation. The result was divided by the product of the number of birds in the group by the number of days. The maximum possible index value calculated using this method is 3.0. 
Allantoic fluid clarified by low-speed centrifugation was diluted with PBS to 128 HAU. To $250 \mu \mathrm{L}$ of the obtained viral sample, $50 \mu \mathrm{L}$ of $2.5 \%$ chicken erythrocytes diluted in the same buffer were added and incubated, periodically shaking, at $+4^{\circ} \mathrm{C}$ for $1 \mathrm{~h}$. The erythrocytes with the virus adsorbed on them were centrifuged at $2800 \mathrm{rpm} / \mathrm{min}$ for $1 \mathrm{~min}$ at $+4{ }^{\circ} \mathrm{C}$, the supernatant was removed, and $250 \mu \mathrm{L}$ of 0.1 M MES buffer with a pH in the range from 4.5 to 6.0 was added. After that, samples were incubated with shaking for $1 \mathrm{~h}$ at $37^{\circ} \mathrm{C}$. Untreated erythrocytes without virus served as a negative control, and erythrocytes with the addition of $0.5 \%$ Tween- 20 served as a positive control. After incubation, the samples were centrifuged for $1 \mathrm{~min}$ at $2800 \mathrm{rpm}, 170 \mu \mathrm{l}$ of the supernatant was transferred to a flat-bottom 96-well plate for measuring optical density at a wavelength of $415 \mathrm{~nm}$ using an iMark Microplate Reader (BioRad, USA). Based on the measurement results, a graph was constructed on which the value of the $\mathrm{pH}$-dependent conformational change of HA was determined.

\subsection{Determination of virus affinity with the receptor analogue - fetuin}

The viruses' affinity for the peroxidase-labelled fetuin was determined in a direct solid phase binding assay [22]. [Matrosovich et al, 2000]. In brief, $100 \mathrm{mkl}$ of IAFs were added to each well of the fetuin-coated microplates. After incubation at $4^{\circ} \mathrm{C}$ overnight, the plates were washed with ice-cold washing buffer (0.02\% Tween 80 in PBS). Serial twofold dilutions of peroxidase-labelled fetuin in the reaction buffer (RB; $0.02 \%$ Tween $80,0.02 \%$ bovine serum albumin, $1 \mathrm{mkM}$ oseltamivir carboxylate in PBS) were added into the wells $(50 \mathrm{mkl} / \mathrm{well})$, and the plates were incubated at $4^{\circ} \mathrm{C}$ for $1 \mathrm{~h}$. After washing, the peroxidase activity in the wells was assayed with tetramethylbenzidine substrate solution. The absorbencies at $450 \mathrm{~nm}$ were determined, the data were converted to Scatchard plots ( $A_{450} / C$ versus $A_{450}$ ), where $C$ is the fetuin concentration expressed in $\mu \mathrm{M}$ sialic acid.

\subsection{Determination of receptor specificity by competitive inhibition}

The association constants of viral complexes with non-labeled sialylglycopolymers (SGPs) were determined in a solid-phase fetuin binding inhibition assay [23]. The viruses were adsorbed in the well of fetuin-coated plates as described above. Serial twofold dilutions of SGPs in solution of peroxidase-labelled fetuin in RB were added into the wells ( $50 \mathrm{mkl} /$ well), and the plates were incubated at $4^{\circ} \mathrm{C}$ for $1 \mathrm{~h}$. After washing, the peroxidase activity in the wells was assayed as described above. The absorbencies at 450 nm were determined, transferred to a PC and processed using Microsoft Excel software.

\subsection{Infection of mice}

Six week-old BALB/c mice were used. Groups of mice were anesthetized and inoculated intranasally with IAF or placebo (PBS). Doses of infectious material are indicated in the tables. Survival and body weight following infection were monitored daily. On days 15 post-infection, serum samples were taken from survivor mice for antibody titration.

\subsection{Determination of antibodies against influenza viruses in mouse sera}

The levels of antibody were assessed by ELISA assay with anti-mouse IgG. Plates (Nunc, MaxiSorp) were covered with fetuin. $100 \mathrm{mkl}$ of IAFs were added to each well of the fetuin-coated microplates, kept overnight at $+40 \mathrm{C}$, then washed and blocked with $0.2 \%$ BSA solution in PBS, $1 \mathrm{~h}$. The blocking solution was removed, $100 \mu \mathrm{L}$ of buffer $(0.1 \%$ 
Tween-20, 0.2\% BSA on PBS) was added to the wells, on which the sera were titrated, starting from a dilution of 1:20. Wells without virus were used as blank. Sera from uninfected mice served as negative controls. Incubation was performed for $4 \mathrm{~h}$ at $40 \mathrm{C}$. After washing, peroxidase-labeled antibodies against mouse immunoglobulins (Sigma, USA) were added. It was incubated for $2 \mathrm{~h}$; after washing, a color reaction with tetramethylbenzidine substrate solution was carried out.

\subsection{Foci of virus infection on MDCK culture.}

The MDCK cells were grown in 96-well plates. The cultures were washed, and $200 \mu \mathrm{L}$ of the DMEM (Gibco) medium with $0.1 \%$ BSA were added to the wells. $50 \mu \mathrm{L}$ of IAFs were added to the outermost wells and titrated by transferring $50 \mu \mathrm{L}$ of the solution. After 16 $\mathrm{h}$, glutaraldehyde solution $(20 \mu \mathrm{L})$ was added to the wells to a final concentration of $0.02 \%$, incubated for $30 \mathrm{~min}$; the medium was poured out, and the wells were washed. Solution of chicken antibodies to FPV/Rostock/34 in PBS supplemented with $0.1 \%$ Tween-20, 0.2\% BSA was added, the plates were incubated for $2 \mathrm{~h}$ at $4^{\circ} \mathrm{C}$ and washed. Solution of anti-chicken antibodies conjugated with horseradish peroxidase was added, the plates were incubated for $1 \mathrm{~h}$ at $4^{\circ} \mathrm{C}$ and washed. Infected cells was visualized by incubation with $0.1 \mathrm{ml}$ of substrate solution per well (0.05\% 3-amino-9-ethylcarbazole, $0.01 \% \mathrm{H}_{2} \mathrm{O}_{2}$ in $0.05 \mathrm{M}$ sodium acetate buffer, $\mathrm{pH} 5.5$ ) for $30 \mathrm{~min}$.

\subsection{Statistical analysis}

Statistical significance was determined with GraphPad Prism (Graphpad Software, Inc.) using the Student's t-test. Statistical significance was defined as $\mathrm{P}<0.05$.

\subsection{Molecular models}

Atomic coordinates of H7 HA (1ti8) were obtained from the Protein Data Bank [24]. The molecular model was generated with DS ViewerPro 5.0 software (Accelrys Inc.).

\section{Results}

3.1. Emergence of Mutations during passaging of the laboratory strain A/FPV/Rostock/34 virus through the chicken lungs

Ten passages of the laboratory virus strain A/FPV/Rostock/34 through chickens lungs were carried out. Sequencing of the entire genome of the original and final variants revealed 9 amino acid (a.a.) substitutions between them in PB2, PB1, PA, HA, M2, and NS1 proteins (Table 1). No differences were found in proteins NA, NP, M1, and NS2.

Among these mutations, the Gly140Arg substitution in HA1 is a reversal, since Arg is located at position 140 of HA1 (numbering according to H3) in all derivate strains of the A/FPV/Rostock/34 (H7N1) according to the GenBank data (V01105, GU052946, CY077420, M24457).

Table 1. Mutations emerging during serial passages of the virus A/FPV/Rostock/34 through the chicken lungs.

\begin{tabular}{|l|c|c|c|c|c|c|c|c|c|c|}
\hline \multicolumn{1}{|c|}{ Protein } & PB2 & PB2 & PB1 & PB1-F2 & PA & HA1 & HA2 & M2 & NS1 & NS1 \\
\hline A.a. Position* & 109 & 567 & 621 & 4 & 32 & 140 & 101 & 82 & 112 & 118 \\
\hline 0 passage & Val & Val & Gln & Glu & Trp & Gly & Ala & Ser & Arg & Met \\
\hline 5 passage & Phe & Ala & Lys & Glu & Ala & Gly & Thr & Ser & Arg & Arg \\
\hline 6 passage & Phe & Ala & Lys & Glu & Ala & Arg & Thr & Ser & Arg & Arg \\
\hline 10 passage & Phe & Ala & Lys & Gly & Ala & Arg & Thr & Arg & Lys & Arg \\
\hline
\end{tabular}


* HA amino acids are numbered according to H3.

\subsection{Arg140 location on the hemagglutinin molecule}

Arg140 is adjacent to constant Cys139, which forms a disulfide bridge with constant Cys97, which, in turn, is adjacent to the key amino acid of receptor binding site Tyr98. Arg141 in HA1 is a constant amino acid in viruses of some subtypes. Arg140 is located at the edge of the receptor-binding site, at the apex of the hemagglutinin. Arg140 is unique to the H7 subtype, it is very conservative. The positively charged atoms of the Arg140 amino groups are directly adjacent to the edge of the receptor binding site and can influence binding to the negatively charged sialic acid, the terminal group of the receptor. In addition, the high positive charge of the three Arg140 located in the apical part of the hemagglutinin trimer promotes mutual repulsion of the spikes and can loosen the structure of the virion.

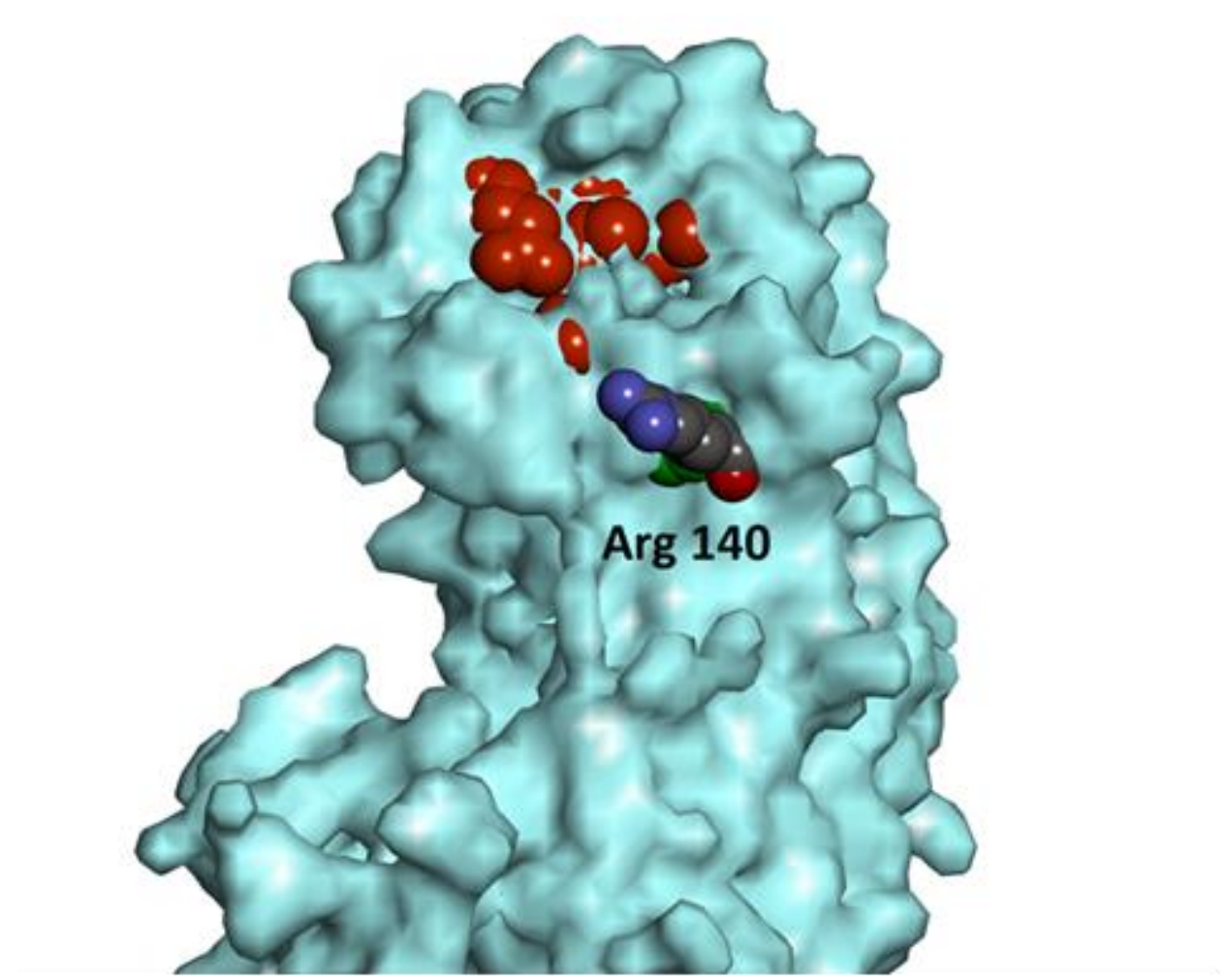

Figure 1. Arg140 location near the hemagglutinin receptor binding site. Atomic coordinates of H7 HA (1ti8) were used [24]. The key amino acids of receptor binding site are colored red.

3.3. Increasing the pathogenicity of $\mathrm{A} / \mathrm{FPV} /$ Rostock/34 variants during passage through the chicken lungs

The original laboratory strain with the Arg140Gly substitution was low pathogenic for chickens. Chicken embryos infected with this virus died within 24-30 hours, nevertheless, infected chicks, even one weeks of age, died rarely. During the passage of this virus through the chicken lungs until the fifth passage, no changes in pathogenicity occurred, but starting from the sixth passage the virus became very pathogenic for chickens. None of the birds infected with variants obtained after the fifth passage survived (Table 2).

Table 2. Survival of one-week-old and eight-week-old chicks after infection with the passage variants of the virus A/FPV/Rostock/34. The data on the apathogenic virus A/mallard/Sweden/91/02 
(H7N9) and the highly pathogenic virus A/chicken/Kurgan/5/2005 (H5N1) are given for comparison.

\begin{tabular}{|l|l|c|c|c|c|}
\hline \multicolumn{2}{|c|}{ Chick age } & \multicolumn{2}{c|}{ one-week-old chicks } & \multicolumn{2}{c|}{ eight-week-old chicks } \\
\hline Variants & designation & infected & survived & infected & survived \\
\hline mallard/Sweden/91/02 & H7N9 & 6 & 6 & 2 & 2 \\
\hline chicken/Kurgan/5/2005 & H5N1 & 5 & 0 & 5 & 0 \\
\hline & & & & & \\
\hline 0 passage & R0p & 4 & 3 & 5 & 5 \\
\hline 1* passage & R1p & 3 & 1 & 2 & 2 \\
\hline 2 passage & R2p & 3 & 2 & 2 & 2 \\
\hline 3 passage & R3p & 3 & 3 & 6 & 6 \\
\hline 4 passage & R4p & 2 & 2 & 2 & 2 \\
\hline 5 passage & R5p & 2 & 2 & 2 & 2 \\
\hline & & & & & \\
\hline 6 passage & R6p & 5 & 0 & 15 & 0 \\
\hline 5 passage & R7p & 2 & 0 & & \\
\hline 6 passage & R8p & 2 & 0 & & \\
\hline 10 passage & R9p & 2 & 0 & & \\
\hline 10 passage & R10p & 2 & 0 & 6 & 0 \\
\hline
\end{tabular}

Since a sharp jump in the pathogenicity occurs between the fifth and sixth passage, we carried out a complete sequencing of these variants (R5p and R6p). In all genes of R5p and R6p, only one amino acid difference was found: replacement Gly140Arg in HA1 (tabl.1)

\subsection{Pathogenicity index of strains R5p and R6p}

Two groups of three 6-week-old chickens were infected intravenous with 10 times diluted IAFs of the R5p and the R6p strains. One of birds threated with the R5p strain died on the day 2 , the second showed signs of the disease until day 10 , and the third was sick until day 4 and recovered. All birds infected with the R6p strain died on the day 2 after infection. The pathogenicity index for these strains was 1.7 and 2.8, respectively. According to the OIE instructions, avian influenza viruses with a pathogenicity index of 1.2 or more (maximum value 3.0 ) are considered highly virulent.

Thus, the R6p strain is a typical highly virulent virus, while the R5p strain retains its highly virulent status, although its pathogenicity was reduced in comparison with the R6p strain.

3.5. Pathogenicity of R5p and R6p for mice, compared with highly pathogenic virus A/chicken/Kurgan/5/2005 (H5N1) and apathogenic virus A/mallard/Sweden/91/02(H7N9).

Mice were infected intranasal with varying doses $\left(10^{1}-10^{6} \mathrm{EID} / \mathrm{mouse}\right)$ of R5p and R6p strains, as well as apathogenic virus A/mallard/Sweden/91/02 (H7N9) and highly pathogenic A/chicken/Kurgan/5/2005 (H5N1), which were taken for comparison. The mice were monitored and weighed daily. The surviving mice, which were reduced in weight within 5 days, were classified as diseased. On the 15th day, antibodies against the infection viruses were determined in all surviving mice. 
Table 3. Effect of Gly140Arg amino acid substitution in HA1 of influenza A/FPV/Rostock/34 on pathogenicity for mice

\begin{tabular}{|l|l|c|c|c|c|}
\hline Virus & Cleavage site of HA & $\begin{array}{c}\text { a.a. in } \\
140 \mathrm{HA} 1\end{array}$ & $\begin{array}{c}\text { Immunogenic } \\
\text { dose }\left(\mathrm{ImD}_{50}\right)\end{array}$ & $\begin{array}{c}\text { Disease dose } \\
\left(\mathrm{DD}_{50}\right)\end{array}$ & $\begin{array}{c}\text { Lethal dose } \\
\left(\mathrm{LD}_{50}\right)\end{array}$ \\
\hline R5p & SKKRKKR | GLF & Gly & $\mathbf{1}$ & $\mathbf{4}$ & $\mathbf{5}$ \\
\hline R6p & SKKRKKR | GLF & Arg & $\mathbf{1}$ & $\mathbf{3}$ & $\mathbf{4}$ \\
\hline H7N9 & PKGR--- | GLF & Arg & $\leq 4$ & $\mathbf{> 6}$ & $>\mathbf{6}$ \\
\hline H5N1 & ERRRKKR | GLF & Pro & $\leq \mathbf{0 , 3}$ & $\mathbf{0 . 3}$ & $\mathbf{0 . 3}$ \\
\hline
\end{tabular}

H7N9 - A/mallard/Sweden/91/02 ; H5N1 - A/chicken/Kurgan/5/2005. The second column shows the structure of the cleavage site. $\mathrm{ImD}_{50}$ is the decimal logarithm of the virus dose, expressed in EID 50 , leading to an immune response in $50 \%$ of mice. DD 50 is the logarithm of the virus dose leading to disease in $50 \%$ of mice. $\mathrm{LD}_{50}$ is the logarithm of the virus dose resulting in the death of $50 \%$ of the mice. The averaged data of three experiments are given.

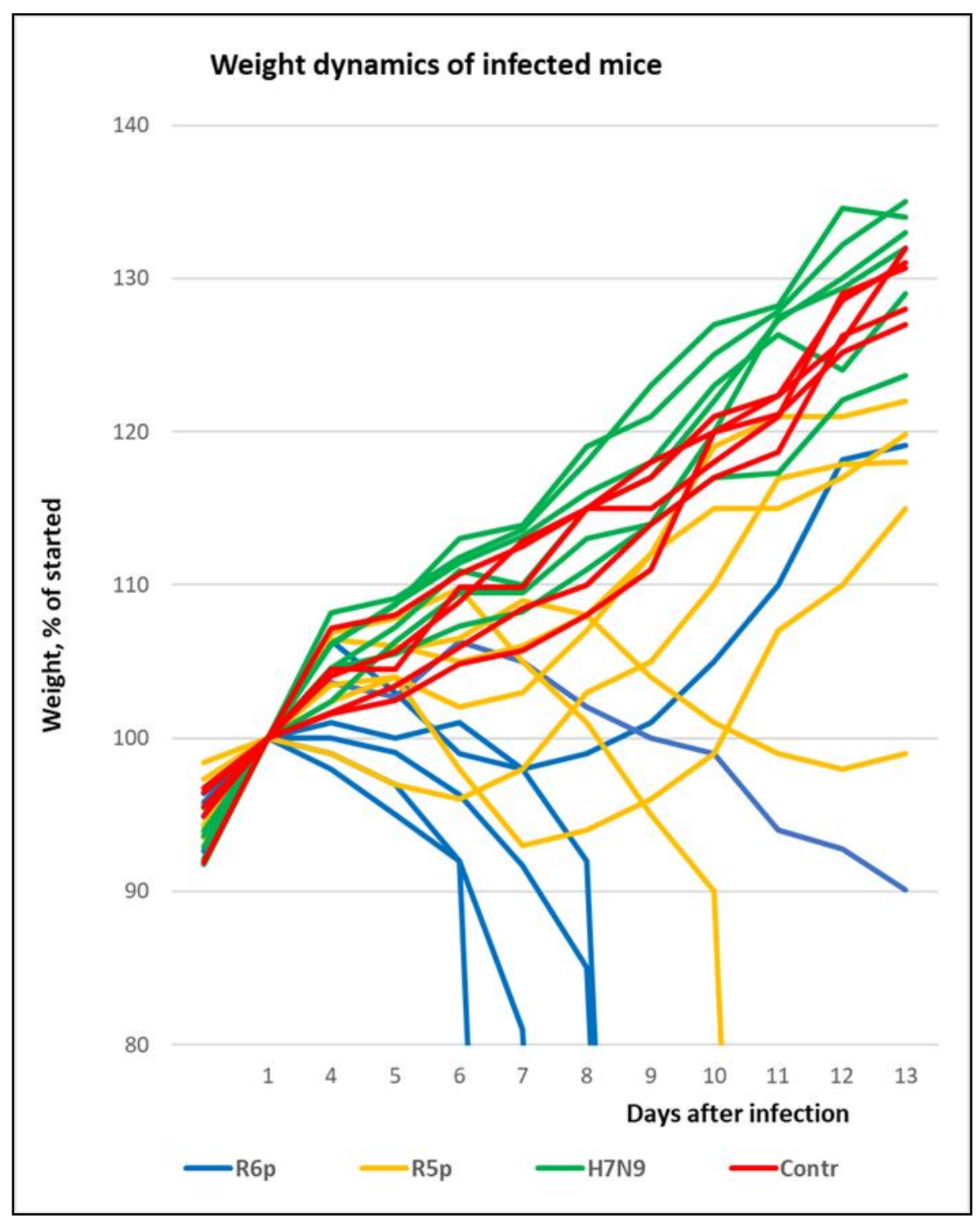

Figure 2. Weight dynamics and survival of mice infected with R5p, R6p and the mallard/Sweden/91/02 (H7N9) virus

In the H5N1 virus, the lethal dose differs little from the immunogenic dose; only occasionally surviving mice with antibodies were detected, and clearly ill and surviving mice were almost never detected. All these doses are close to one infectious unit EID 50 of the 
virus. The mallard/Sweden/91/02 (H7N9) virus caused an asymptomatic infection in mice when challenged at the maximum dose, and elicited a strong immune response.

The pathogenicity of passage variants of the A/FPV/Rostock/34 for mice are between the H7N9 and the highly pathogenic H5N1viruses. Both strains R5p and R6p killed mice when infected with high doses of the virus. The lethal dose of the R6p strain was an order of magnitude lower than that of the R5p strain. When infected with sublethal doses of both strains, a reliable picture of the disease in mice was observed. Lower doses, up to nearly 10 EID50, did not cause disease, but induced an immune response.

The pathogenicity of the studied viruses correlates with the structure of the cleavage site of HA, although in highly pathogenic $\mathrm{H} 7$ and $\mathrm{H} 5 \mathrm{~N} 1$ viruses it can be determined by other factors associated with other genes [25]. Substitution Gly140Arg undoubtedly increases the pathogenicity of the virus, since this is the only difference between the R5p and R6p strains.

The typical experiment when mice were infected with $10^{4}$ EID 50 the three above-mentioned H7 viruses is shown on Figure 2. 1/6 and 4/6 mice infected with R5p and R6p, respectively, died, while mice infected with the A/mallard/Sweden/ 91/02 virus suffered an asymptomatic infection. All surviving mice were found to have antibodies to H7 HA.

3.6. Effect of Gly140Arg substitution on the $\mathrm{pH}$-dependent conformational change of HA.

One of the factors influencing the pathogenicity of influenza viruses is the value of $\mathrm{pH}$ dependent conformational change of HA. In viruses of wild ducks, the conformational change occurs at $\mathrm{pH}$ close to five. A shift in the $\mathrm{pH}$ transition towards less acidic $\mathrm{pH}$ is characteristic of highly virulent strains of poultry influenza viruses. During attenuation of the highly pathogenic virus A/chicken/Kurgan/5/2005 (H5N1) a sharp drop in the transition $\mathrm{pH}$, due to mutations in the stem part of HA, was shown [26]. It has been shown that a high $\mathrm{pH}$ transition increases the tropism of viruses to human endothelial cells [27]. We compared the transition $\mathrm{pH}$ of strains R5p and R6p to test the hypothesis that substitution increases the pathogenicity of the virus by increasing the $\mathrm{pH}$ of transition. However, this hypothesis was not confirmed. As can be seen from Figure 3, the curves of $\mathrm{pH}$-dependent erythrocyte hemolysis for strains R5p and R6p practically coincide, that is, the Gly140Arg substitution did not affect the value of the $\mathrm{pH}$-dependent conformational change of the FPV/Rostock/34 virus. Obviously, this is due to the surface arrangement of Arg140. 


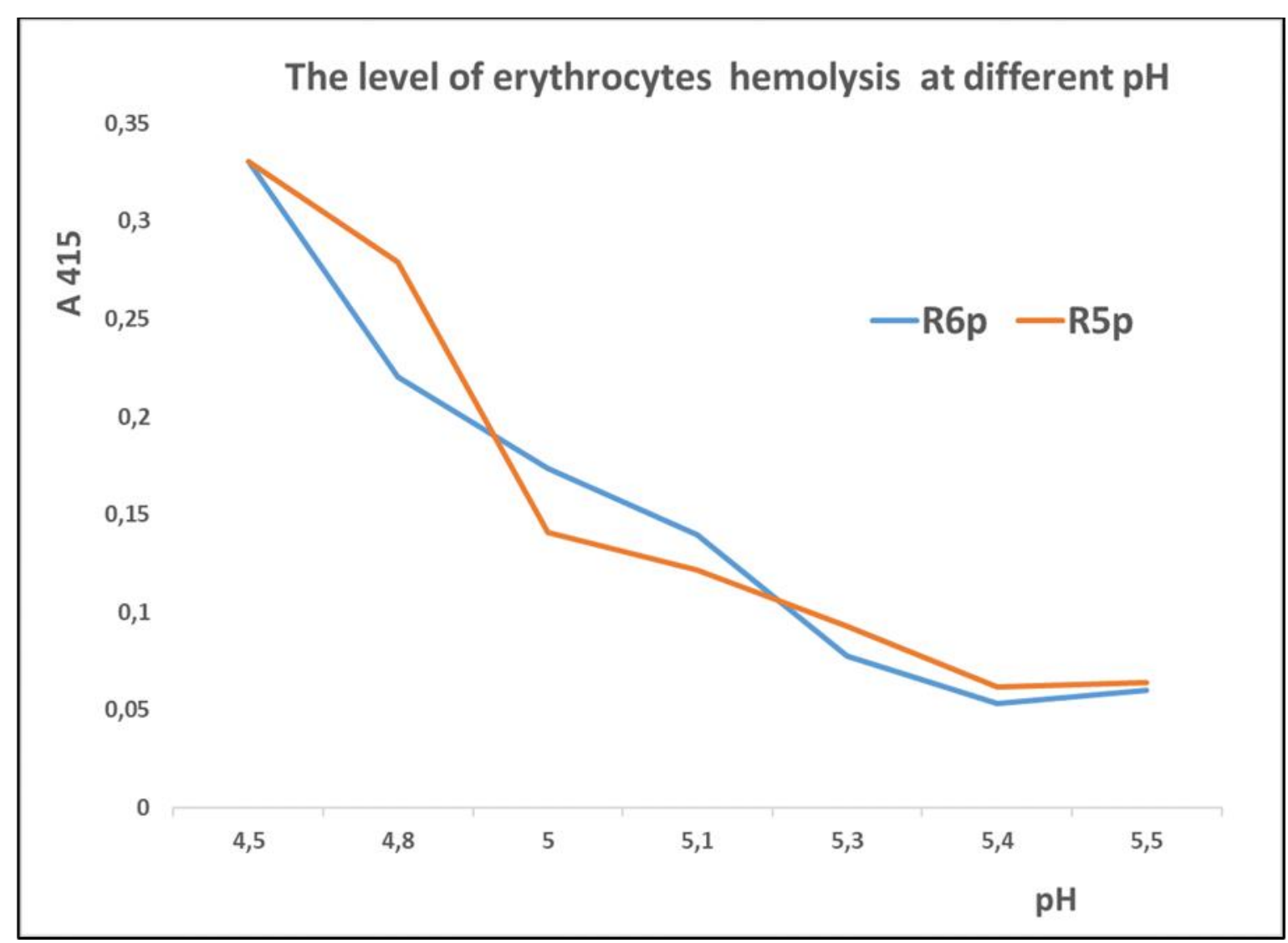

Figure 3. The $\mathrm{pH}$ dependence of erythrocyte hemolysis in complex with R5p and R6p viruses. A415 - absorption at 415 $\mathrm{nm}$, which corresponds to the degree of erythrocyte hemolysis.

3.6. Effect of charge mutation Gly140Arg in hemagglutinin on the affinity for receptor analogs and on receptor specificity. 


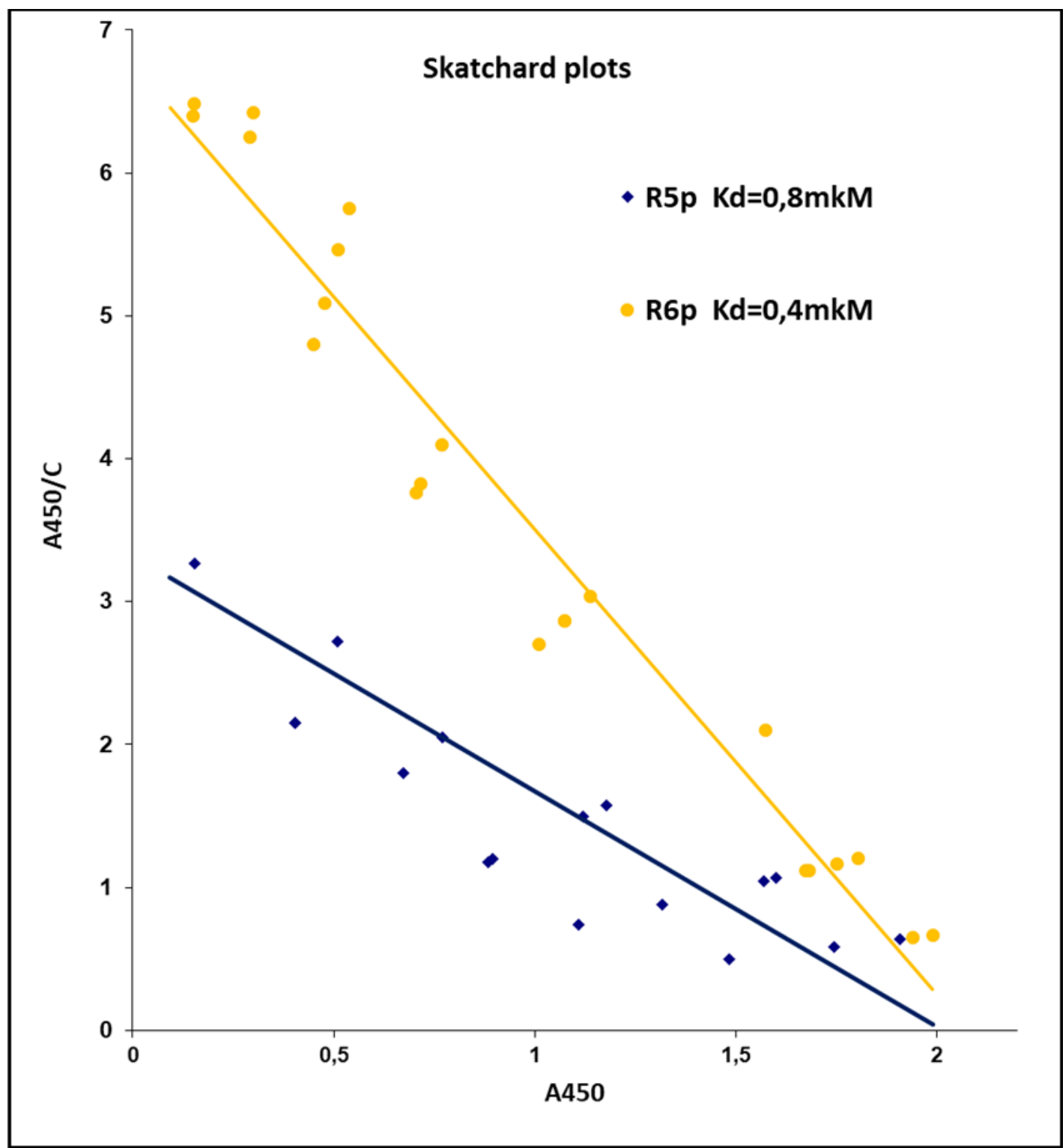

Figure 3. Affinity of peroxidase labeled fetuin for virions of R5p and R6p strains. The results of testing Fet-HRP on a plate coated with R5p and R6p viruses are presented in Scatchard coordinates. The X-axis is the A450 signal reflecting the amount of bound fetuin, and the $\mathrm{Y}$-axis is the ratio of the A450 signal to the fetuin concentration (expressed in mkM of sialic acid) in the solution.

Fetuin - fetal bovine serum albumin - is a glycoprotein that contains bi- and dendritic Nglycans ending in Sia $\alpha 2-3 \mathrm{Gal} \beta 1-4 \mathrm{GlcNAc-}$ and Sia $\alpha 2-6 \mathrm{Gal} \beta 1-4 \mathrm{GlcNAc}$ motifs [28]. This makes it a good model receptor for most influenza viruses. We compared the affinity of strains R5p and R6p for fetuin labeled with horseradish peroxidase. The analysis of FetHRP binding to virions adsorbed on 96 well panels was performed in Scatchard coordinates (Fig. 4). The affinity of fetuin for the R6p virus is two times higher than for the R5p virus (Dissociation constants are $0.4 \mu \mathrm{M}$ and $0.8 \mu \mathrm{M}$, respectively). This is probably due to the strong negative charge of fetuin and a sharp increase in the positive charge in the R6p strain compared to R5p due to the replacement of Gly by Arg.

3.7. Comparison receptor specificity patterns of viruses R5p and R6p. 
Receptor specificity of the viruses was characterized in fetuin-binding inhibition assay using synthetic sialylglycopolymers, attached to the polymer [29]. The structures and designations of the oligosaccharide moieties are shown below.

Table 4. Structures of oligosaccharides attached to polyacrylamide.

\begin{tabular}{|c|c|}
\hline Sialyloligosaccharide & designation \\
\hline Neu5Ac $\alpha 2-6$ Gal $\beta 1-4 G l c N A c \beta-$ & 6'SLN \\
\hline Neu5Ac $\alpha 2-3$ Gal $\beta 1-4 G 1 c \beta-$ & 3'SL \\
\hline Neu5Ac $\alpha 2-3$ Gal $\beta 1-4 G l c N A c \beta-$ & 3`SLN \\
\hline Neu5Ac $\alpha 2-3$ Gal $\beta 1-4-(6-O-S u) G l c N A c \beta-$ & Su3`SLN \\
\hline Neu5Ac $\alpha 2-3$ Gal $\beta 1-4($ Fuc $\alpha 1-3)$ GlcNAc $\beta-$ & SLe $^{\mathrm{x}}$ \\
\hline Neu5Ac $\alpha 2-3$ Gal $\beta 1-3$ GlcNAc $\beta$ - & $\mathrm{SLe}^{\mathrm{c}}$ \\
\hline Neu5Ac $\alpha 2-3$ Gal $\beta 1-3$ (Fuc $\alpha 1-4)$ GlcNAc $\beta-$ & $\mathrm{SLe}^{\mathrm{a}}$ \\
\hline Neu5Ac $\alpha 2-3$ Gal $\beta 1-3$ GalNAc $\alpha-$ & STF \\
\hline Neu5Ac $\alpha 2-3$ Gal $\beta 1-3-(6-S u) G a l N A c \alpha-$ & Su-STF \\
\hline
\end{tabular}

An example of determining the receptor specificity patterns of viruses is shown in Fig. 5 .

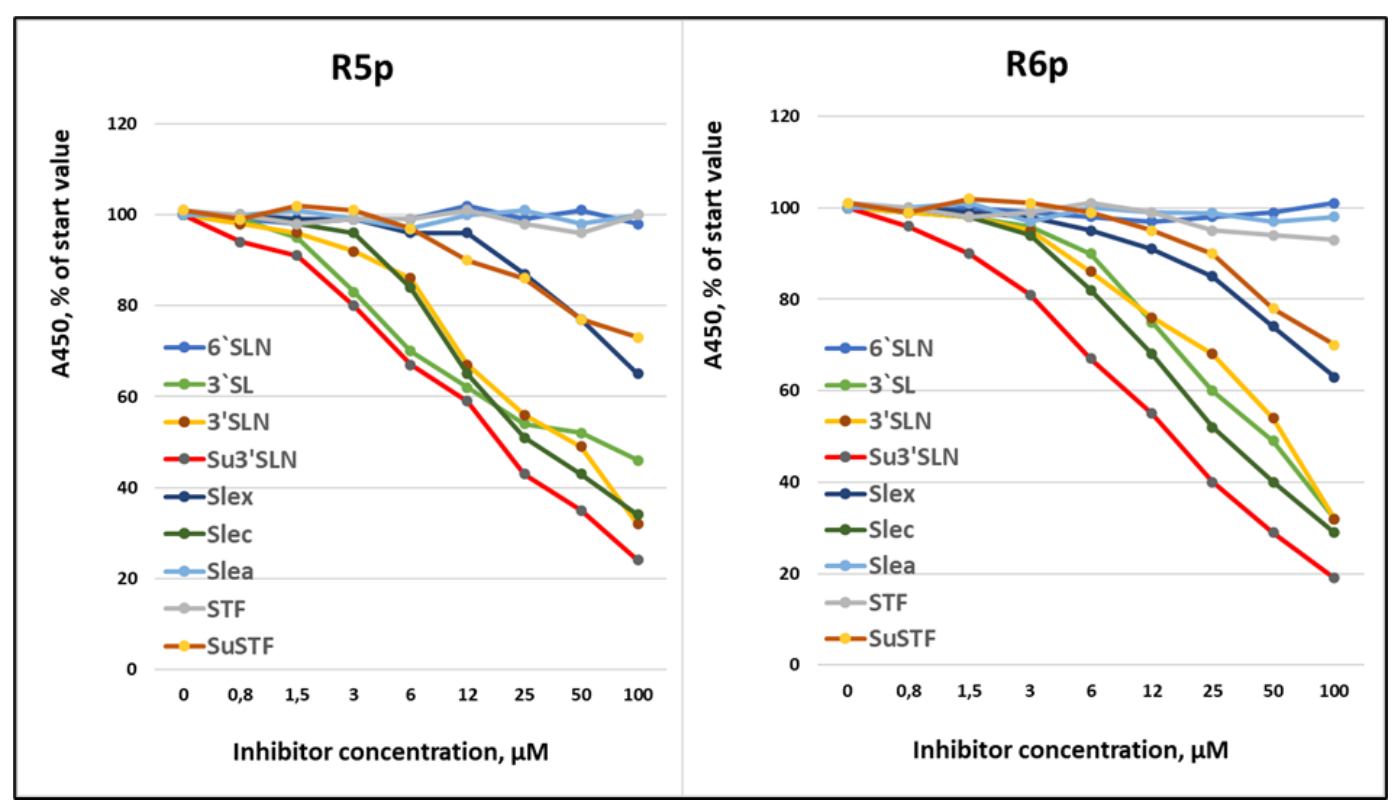

Figure 5. Inhibition of binding of labeled fetuin to viruses R5p and R6p by the receptor analogs. Receptor analogs were added to a solution of labeled fetuin at the indicated concentrations and the level of binding of the label to the virus was determined.

Receptor determinants 3'SL, 3'SLN, Slec and Su-3'SLN reliably inhibit binding to both strains (R5p and R6p), while 6 SLN, SLea and STF do not inhibit. R5p and R6p showed similar binding profiles, typical for avian viruses of the H7 subtype [2]. The similarity of the binding patterns of the R5p and R6p viruses indicates that the Gly140Arg substitution in HA1 did not affect the interaction of the receptor-binding site of the virus with the receptor. 
3.8. Foci of the infected cells produced by the viruses in MDCK monolayer.

The assay used was based on the method of cultivation influenza viruses in MDCK cells in 96-well microplates and detection of foci of infected cells by immunoperoxidase staining [30]. Infected cells were treated with chicken antibodies against FPV/Rostock/34 virus followed to peroxidase labeled anti-chicken antibodies and stained with aminoethylcarbazole - hydrogen peroxide solution. Cells infected with the virus acquired a red color.

The R5p produces large indistinct foci, which bear gaps of noninfected cells inside the focus. The foci of R6p are small, round-shaped and reveal no gaps of noninfected cells between the stained cells surrounding the center of the focus. These features of the foci suggest that the R6p virus progeny spreads directly from cell to cell, while R5p virus progeny released into the culture medium and infect the distance cells. The difference shape of foci produced by R5p and R6p viruses suggests that affinity of R6p to cells is higher than affinity of R5p.

A

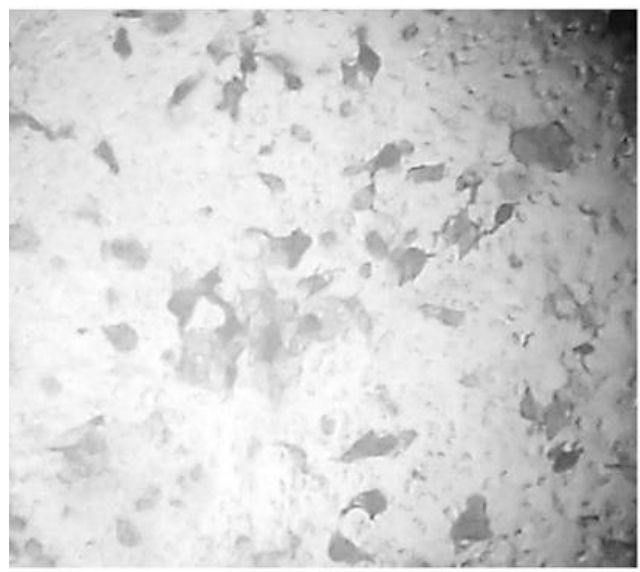

B

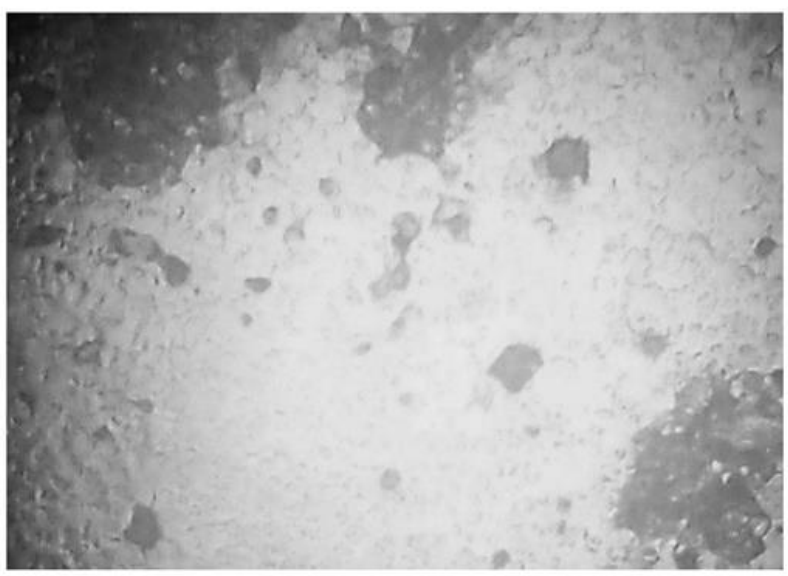

Figure 6. Different pictures of cells infected with R5p (A) and R6p (B) variants of influenza virus FPV/Rostock/34.

Monolayer of MDCK cells was infected influenza FPV/Rostock/34 R5p and R6p, incubated 16 h and stained as described in material and method section.

\section{Discussion}

The viruses with H7 HA subtypes were isolated from a wide range of hosts such as wild ducks, poultry, horses, seal and humans. They caused disease outbreaks in poultry ("fowl plaque") in both hemispheres. Little genetic diversity has been observed between $\mathrm{H} 7$ viruses isolated from wild birds and poultry birds in the same time and region suggesting their unrestricted interspecies transmission.

Such a wide range of hosts may be because that modern H7 AIVs are descendants of viruses, which long ago had transmitted from ducks to chickens, adapted to a new host, and then returned to wild ducks again, which allows the virus to persist and spread. This is confirmed by the topology of the evolutionary trees of $\mathrm{H7}$ viruses, at the base of which not duck, but chicken viruses are grouped [2].

The receptor specificity of H7 AIVs also has a dual character. They recognize the chicken receptors - Su3'SLN and Su-Slex as well as the main duck receptor Slec. Many H7 
AIVs also show some affinity for the "human" receptor, 6'SLN. Naturally, this broad receptor specificity contributes to the ability to infect a wide range of hosts. The structure of the receptor-binding site of hemagglutinin $\mathrm{H} 7$ indicates the rearrangement that occurred during adaptation to the new receptor in new hosts. All H7 AIVs have Lys193 in HA, similar to HPAIV H5N1. Lys193 provides increased binding of viruses to sulfated receptors Su-3'SLN and Su-SLex due to electrostatic interaction of the sulfate residue of the receptor with the positively charged lysine side chain [23]. The set of amino acids 185-189 located at the bottom of the RBS is unique [2]. The pair Arg140 and Arg141 is another feature of H7 HA. Arg141 in HA1 is a constant amino acid in viruses of H7, H15, H10, $\mathrm{H} 14, \mathrm{H} 3$ and $\mathrm{H} 4$ subtypes. It plays an important role in maintaining the structure of the PBS, since the Arg141 residue is inside the molecule and it's positively charged groups contact with negatively charged oxygen Asp77, Gly72, and Phe147 [24]. Arg140 is unique to the $\mathrm{H7}$ subtype; it is located at the edge of the receptor-binding site, at the apex of the hemagglutinin. Arg140 is very conservative in H7 HA, although in rare cases it replaced by Lys or a small amino acid.

Reversion of Gly140Arg dramatically increases the surface positive charge of virions and promotes the binding of the virus to the negatively charged surface and molecules. R6p strain differs from R5p by an increased pathogenicity for chickens and mice, increased affinity for a negatively charged receptor analogue, an increased affinity for MDCK cells. In the same time, a pattern of receptor specificity and the $\mathrm{pH}$ of the hemagglutinin transition of the R6p do not change. The increased pathogenicity of R6p for chickens and mice is probably due to an increased affinity for negatively charged host cells and receptors.

Author Contributions: Anastasia Treshchalina, Yulia Postnikova, Alexandra Gambaryan, conceived and designed the experiments; Anastasia Treshchalina, Yulia Postnikova, Elizaveta Boravleva, Galina Sadykova, Alexandra Gambaryan, and Natalia Lomakina performed the experiments; Alexandra Gambaryan, Alexei Prilipov and Natalia Lomakina analyzed the data; Alexandra Gambaryan and Anastasia Treshchalina wrote the paper.

Funding: This research was funded by the research grant 17-04-00148 from the Russian Foundation for Basic Research. Publication was supported by Chumakov Federal scientific center for the research and development of immune-and-biological products.

Institutional Review Board Statement: The study was conducted according to the guidelines of European Convention for the Protection of Vertebrate Animals used for Experimental and Other Scientific Purposes, Strasbourg, 18 March 1986. The study design was approved by the Ethics Committee of the Chumakov Federal scientific center for the research and development of immune-and-biological products, Moscow, Russia (Approval \#4 from 2 December 2014).

Acknowledgments: We thank Dr. R. Fouchier (Department of Virology, Erasmus Medical Center, the Netherlands) for providing non-pathogenic mallard virus A/mallard Sweden 91/02 (H7N9) and Professor N. V. Bovin and members of the Laboratory of Carbohydrates (Shemyakin and Ovchinnikov Institute of Bioorganic Chemistry, Russian Academy of Sciences, Moscow) for providing analogues of cellular receptors.

Conflicts of Interest: The authors declare no conflict of interest.

\section{References}

1. Webster, R.G. Influenza virus: transmission between species and relevance to emergence of the next human pandemic. Arch Virol Suppl 1997, 13, 105-113.

2. Gambaryan, A.S., Matrosovich, T.Y., Philipp, J., Munster, V.J., Fouchier, R.A., Cattoli, G., Capua, I., Krauss, S.L., Webster, R.G., Banks, J., Bovin, N.V., Klenk, H.D., Matrosovich, M.N. Receptor-binding profiles of H7 subtype influenza viruses in different host species. J. Virol. 2012, 86, 4370-4379. 
3. Scheibner, D., Ulrich, R., Fatola, O.I., Graaf, A., Gischke, M., Salaheldin, A.H., Harde,r T.C., Veits, J., Mettenleiter, T.C., Abdelwhab, E.M. Variable impact of the hemagglutinin polybasic cleavage site on virulence and pathogenesis of avian influenza H7N7 virus in chickens, turkeys and ducks. Sci Rep. 2019, 9(1), 11556. doi: 10.1038/s41598-01947938-3.

4. Davison, S., Eckroade, R. J., Ziegler, A. F. A Review of the 1996-98 Nonpathogenic H7N2 Avian Influenza Outbreak in Pennsylvania. Avian Dis. 2003, 47, 823-827.

5. Selleck, P.W., Arzey, G., Kirkland, P.D. et al. An Outbreak of Highly Pathogenic Avian Influenza in Australia in 1997 Caused by an H7N4 Virus. Avian Dis. 2003, 47, 806-811.

6. Capua, I., Marangon, S. The avian influenza epidemic in Italy (1999-2000): a review. Avian Pathol. 2000, $29,289-294$.

7. Munster, V.G., de Wi,t E., van Riel, D. et al. The molecular basis of the pathogenicity of the Dutch highly pathogenic human influenza A H7N7 viruses. J. Infect. Dis. 2007, 196(2), 258-265.

8. Gao,R., Cao, B., Hu, Y., et al. Human infection with a novel avian-origin influenza A (H7N9) virus. N Engl J Med 2013, 368, 1888-97.

9. Zhumatov, K.Kh., Sayatov, M.Kh., Kydyrmanov, A.I. Influenza A/H7 viruses, distribution, genetic variability, pathogenicity for birds and humans. Eurasian Journal of Applied Biotechnology 2013, 2,12-16. DOI: 10.11134/btp.2.2013.2

10. Bisset, A.T., Hoyne, G.F. Evolution and Adaptation of the Avian H7N9 Virus into the Human Host. Microorganisms. 2020, 8(5), 778. doi, 10.3390/microorganisms 8050778 .

11. Wu, X., Xiao, L., Li L. Research progress on human infection with avian influenza H7N9. Front Med. 2020, 14(1), 820. doi, 10.1007/s11684-020-0739-z.

12. Des Rochers, B., Chen, R.E., Gounder, A.P., Pinto, A.K., Bricker, T., Linton, C.N., Rogers, C.D., Williams, G.D., Webby, R.J., Boon, A.C.M. Residues in the PB2 and PA genes contribute to the pathogenicity of avian H7N3 influenza A virus in DBA/2 mice. Virology. 2016, 494, 89-99. doi, 10.1016/j.virol.2016.04.013.

13. Dreier, C., Resa-Infante, P., Thiele, S., Stanelle-Bertram, S., Walendy-GnirßK., Speiseder, T., Preuss, A., Müller, Z., Stech, J., Klenk, H-D., Gabriel, G. Mutations in the H7 HA and PB1 genes of avian influenza a viruses increase viral pathogenicity and contact transmission in guinea pigs. Emerg Microbes Infect 2019, 8(1), 1324-1336. doi, 10.1080/22221751.2019.1663131.

14. Feldmann, A., Schäfer, M. K., Garten, W, Klenk, H. D. Targeted Infection of Endothelial Cells by Avian Influenza Virus A/FPV/Rostock/34 (H7N1) in Chicken Embryos. J Virol. 2000, 74(17), 8018-8027. doi, 10.1128/jvi.74.17.80188027.2000.

15. Wagner, R., Gabriel, G., Schlesner, M., Alex, N., Herwig, A., Werner, O., Klenk H.-D. Protease Activation Mutants Elicit Protective Immunity Against Highly Pathogenic Avian Influenza Viruses of Subtype H7 in Chickens and Mice. Emerg Microbes Infect, 2013, 2(2), e7. doi, 10.1038/emi2013.7.

16. Schuy, W., Will, C., Kuroda, K., Scholtissek, C., Garten, W., and Klenk, H.-D. Mutations Blocking the Transport of the Influenza Virus Hemagglutinin Between the Rough Endoplasmic Reticulum and the Golgi Apparatus. EMBO J. 1986, 5, 2831-2836.

17. Garten, W., Will, C., Buckard, K., Kuroda, K., Ortmann, D., Munk, K., Scholtissek, C., Schnittler, H., Drenckhahn, D., Klenk, H.-D. Structure and assembly of hemagglutinin mutants of Fowl Plague Virus with impaired surface transport. $J$. Virol. 1992, 66(3), 1495-1505.

18. McCauley, J.W., Penn, C.R. The Critical Cut-Off Temperature of Avian Influenza Viruses. Virus Res. 1990, 17(3), 191-198. doi, 10.1016/0168-1702(90)90064-i.

19. Stech, J., Stech, O., Herwig, A., Altmeppen, H., Hundt, J., Gohrbandt, S., Kreibich, A., Weber, S., Klenk, H.D., Mettenleiter, T.C. Rapid and reliable universal cloning of influenza A virus genes by target-primed plasmid amplification. Nucleic Acids Res. 2008, 36(21), e139. doi, 10.1093/nar/gkn646. 
20. Li, O.T., Barr, I., Leung, C.Y., Chen, H., Guan, Y., Peiris, J.S., Poon, L.L. Reliable universal RT-PCR assays for studying influenza polymerase subunit gene sequences from all 16 haemagglutinin subtypes. J. Virol. Methods. 2007, 142(1-2), 218-222. doi, 10.1016/j.jviromet.2007.01.015.

21. Avian influenza (infection with avian influenza viruses). OIE. Manual of Diagnostic Tests and Vaccines for Terrestrial Animals. 2016, 2, Chap. 2.3.4. - URL, http,

//www.oie.int/fileadmin/Home/eng/Health_standards/tahm/2.03.04_AI.pdf.].

22. Matrosovich, M., Tuzikov, A., Bovin, N., Gambaryan A., Klimov A., Castrucci M. R., Donatelli I., and Kawaoka Y. Early alterations of the receptor-binding properties of $\mathrm{H} 1, \mathrm{H} 2$, and $\mathrm{H} 3$ avian influenza virus hemagglutinins after their introduction into mammals. J. Virol. 2000, 74, 8502-8512.

23. Gambaryan, A.S., Tuzikov, A.B., Pazynina, G.V., Desheva, J.A., Bovin, N.V., Matrosovich, M.N., Klimov, A.I. 6-sulfo sialyl Lewis $\mathrm{X}$ is the common receptor determinant recognized by H5, H6, H7 and H9 influenza viruses of terrestrial poultry. Virol. J. 2008, 5, 85 .

24. Russel, R.J., Gamblin, S.J., Haire, L.F., Stevens, D.I, Xiao, B., Ha, Y., Skehel, J.J. H1 and H7 influenza haemagglutinin structures extend a structural classification of haemagglutinin subtipes. Virology 2004, 325, 287.

25. Fukuyama, S., Kawaoka, Y. The pathogenesis of influenza virus infections, the contributions of virus and host factors. Curr Opin Immunol. 2011, 23(4), 481-486. doi, 10.1016/j.coi.2011.07.016.

26. Lomakina, N.F., Boravleva, E.Y., Kropotkina, E.A., Yamnikova ,S.S., Drygin, V.V., Gambaryan, A.S., Attenuation of A/chicken/Kurgan/3/2005 (H5N1) Influenza Virus Using Selection in an Environment Simulating the Life Cycle of Wild Duck Viruses. Molecular Genetics, Microbiology and Virology, 2011, 26(3), 132-139.

27. Hensen, L., Matrosovich, T., Roth, K., Klenk, H.D., Matrosovich, M. HA-Dependent Tropism of H5N1 and H7N9 Influenza Viruses to Human Endothelial Cells Is Determined by Reduced Stability of the HA, Which Allows the Virus To Cope with Inefficient Endosomal Acidification and Constitutively Expressed IFITM3. J. Virol. 2019, 94(1), e0122319. doi, 10.1128/JVI.01223-19.

28. Hayase, T., Rice, K.G., Dziegielewska, K.M., Kuhlenschmidt, M., Reilly, T. and Lee Y.C. Comparison of N-glycosides of fetuins from different species and human alpha 2-HS-glycoprotein, Biochemistry 1992, 31, 4915-4921.

29. Matrosovich, MN, Gambaryan, AS. Solid-phase assays of receptor-binding specificity. Methods Mol Biol. 2012, 865:7194. doi: 10.1007/978-1-61779-621-0_5

30. Okuno, Y., Tanaka, K., Baba, K., Maeda, A., Kunita, N., and Ueda, S. Rapid focus reduction neutralization test of influenza A and B viruses in microtiter system. J. Clin. Microbiol. 1990, 28, 1308-1313. 